\title{
Incidencia de los factores de variación en las especies de camélidos y tipos domésticos especializados en el NOA. Un paso más allá de la taxonomía en la explicación del proceso de domesticación
}

María del Carmen Reigadas ${ }^{1}$

\begin{abstract}
RESUMEN
Dentro del estudio de la detección de domesticación animal a partir del análisis de fibras arqueológicas, no todas las variaciones fenotípicas se deben a este proceso siendo guiadas selectivamente. Entendiendo que diversas fuentes de variación inciden en los rangos asumidos para los diferentes taxones, en este trabajo se tomará en cuenta: 1) Variabilidad interna para cada especie (edad, sexo, región corporal); 2) Variabilidad de las especies domesticadas en relación al uso económico (lana, carne, transporte); 3) Variabilidad en relación al medio. La información proviene de poblaciones actuales de camélidos domésticos, con las cuales armamos los patrones comparativos en relación con las diferentes fuentes enunciadas. Metodológicamente la ampliación de la muestra por sexo, edad y regiones corporales para cada especie y tipo con distribución ambiental diferenciada confirma que las designaciones para las muestras arqueológicas que responden al patrón doméstico o "tercer grupo" caen dentro de la normal variación de Lama glama, y que ninguno de sus rangos se superpone con formas silvestres. Se observaron diferencias en la composición de los pelajes, por lo que la metodología es apropiada para buscar este tipo de información, permitiendo caracterizar los "tipos" existentes en dos áreas consideradas. En un nivel explicativo, esta determinación es válida para el estudio de patrones ganaderos productivos. La relación con el registro arqueológico indicaría que la selección cultural opera sobre un tipo generalizado de camélido semejante al "tipo intermedio" definido, que promueve a posteriori un patrón productivo especializado en la obtención de carne y fibra.
\end{abstract}

\section{ABSTRACT}

Within the study of animal domestication, it is consider that not all phenotypical variations in archaeological fibers are the result of culturally guided selection. Based on the assumption that different sources on variation influence the ranges adopted by various taxa, this paper considers: 1) Internal variation por each species (age, sex, body region); 2) Variation of domesticaded species in relation to their economical use (wool, meet, transport); 3) Variation in relation to the environment. Data correspond modern domestic camelid population with which we set up comparatived standards in relation to each source

1 Instituto de Biología de la Altura, Universidad Nacional de Jujuy, San Salvador de Jujuy, ARGENTINA. mentioned above. Methodologically, expanded the sample for sex, age and body region por each species and "type" with differential environmental distribution, allows us to confirm that the archaeological samples that belong to the domestic standard or "third group" fall within the normal ranged variation of Lama glama, and none of their ranges overlap with wild forms. Differences in fiber composition were observed, suggehing that the methodology applied is appropriated for searching this kind of information, allowing the characterization of the types present in two areas considered. At an explanatory level determination of types is valid for the study of animal productive patterns. The relation with the archaeological record indicates that cultural selection acts uppon a generalized camelid type similar to the "intermediate type" defined, that latten promotes a productive pattern specialized in the extraction of meet and fiber.

\section{Introducción}

Dentro de una continuidad biológica y cultural el proceso de evolución cultural es visto como la continuación del proceso general de adaptación (Dunnel 1980).

La domesticación animal (control cultural-reproductivo) como producto de la evolución cultural es un fenómeno concreto de transmisión genético-cultural, que crea diversidad por especiación y diferenciación y tipos como resultado de la percepción del beneficio que motoriza el proceso en sí mismo. El objetivo último es la explicación de este proceso (variabilidad/cambio) y su valor adaptativo. Para esto nos planteamos dos grupos de preguntas como punto de partida: 1) En relación a la posibilidad de identificación de formas silvestres y domésticas y la posibilidad de detectar cambios morfológicos de las primeras a las segundas; 2) Sobre cómo podemos describir el proceso ajustando diferencias morfológicas en relación a los diferentes usos, con el fin de resolver por qué se produce el cambio en las sociedades cazadoras recolectoras y cuándo se 
consolidan las sociedades productoras de alimentos en sus diferentes adaptaciones tomando en consideración el segmento social pastoril.

No es nuestro objetivo transferir principios biológicos al estudio de la variación tal como es vista en el registro biológico, sino, como explicitan O'Brien y Holland (1990), explicar mecanismos evolutivos que afecten los cambios en las frecuencias de variación.

La especiación y diferenciación crea diversidad, aunque en este proceso no se crea información genética nueva, sino que se particiona la misma en entidades discretas. La conformación de éstas traducida en variaciones morfológicas permiten reconocerlas a través de rasgos cuantitativos y cualitativos, y particiones en sus componentes genéticos y ambientales (fenotipos).

Entendemos la variabilidad como la potencial variación a producirse y el cambio como sujeto de la evolución en un sentido de proceso selectivo como lo expresa Darwin (1859) y no transformacional. Dentro de la teoría general evolutiva este autor propone un primer paso de generación de variación dentro de un pool fenotípico. Esta ocurre primero a nivel de genotipo. La producción de variación es indirecta y la selección no requiere ninguna fuerza activa. Este proceso de especiación a partir de la selección natural llega a separar poblaciones en unidades evolutivas independientes, acumulando diferencias genéticas. Y la adaptación es vista como el éxito de un organismo resultado de la selección que actúa sobre la variación.

Es en un segundo paso a nivel de fenotipo que la selección activa opera.

Con la domesticación, la producción de cambios genéticos hace que los organismos estén mejor adaptados a las condiciones determinadas por las sociedades humanas (Simmons 1992). Los rasgos seleccionados culturalmente tienen un valor adaptativo si son exitosos funcionalmente en términos de algún beneficio económico para el hombre. La transmisión cultural (a partir de selección cultural) en términos lamarckianos es una fuerza que direcciona el pool de variación que permite la adaptación.

En el caso de efectuarse una selección dirigida estamos frente a la intención humana que introduce la variación.
Para los biólogos una propiedad de los seres vivos es que ellos aparecen en múltiples formas, son variables, diversos (Solbrig 1991). Los cambios producidos en ellos tienen efectos visibles y la diversidad es resultado de mutaciones génicas.

O'Brien y Holland (1990) dicen que en los sistemas culturales las variaciones resultan en parte de las diferencias en la percepción e intención humanas. Aparece entonces un mecanismo de transmisión de caracteres a través del comportamiento como presión selectiva.

Pero ya sea la transmisión genética o cultural, si la variación del rasgo es heredable por algún medio, éste va a tener una representación diferencial en las generaciones siguientes. Entonces ¿cómo podemos identificar el agente de selección que favorece la aparición de determinados rasgos (funcionales en términos adaptativos)?

Volviendo a la domesticación no todas las variaciones registradas se deben a este proceso y son guiadas selectivamente. En estas circunstancias las fuentes de variación son un tema separado del modo en que se opera la selección.

En una primera etapa a partir del estudio morfológico de vellones de camélidos actuales hemos registrado variaciones que traducidas en cambios en los fenotipos han servido como base para nuestras agrupaciones y designaciones taxonómicas para los registros arqueológicos, por comparación con estos homólogos actuales (Reigadas 1991Ms, 1992Ms, 1993).

Partiendo de estos antecedentes que han servido para ajustar metodológicamente una vía alternativa para la detección de domesticación, y los problemas planteados sobre el registro de las variaciones encontradas, hemos planteado tres tipos de situaciones que deben ser controladas: 1) Ecuación entre rasgos morfológicos producto del manejo zootécnico: covariancia; 2) Injerencia de las diferentes fuentes de variación; 3) Incidencia ambiental sobre el genotipo.

Estos problemas inciden en general en los rangos asumidos para los diferentes taxones y en particular sobre algunas de las variables seleccionadas.

La variabilidad empírica de una población (fenotipos) se define como una función de las varianzas 
genotípicas y ambientales en relación a la presencia temprana o no de alteraciones de los rasgos.

Sobre las variables seleccionadas (grosor, color, médula, tipo de fibra) se tomaron en cuenta los patrones de herencia particulares para ver cuáles de ellas se alteran por el impacto ambiental (Reigadas 1993) y cómo pueden ser afectadas en su transmisión y aparición en el fenotipo. Sobre esta situación de control genético se superpone la incidencia de las fuentes de variación internas y externas.

En este trabajo consideraremos el punto 2) antedicho que se compone de variabilidad: 1) interna para cada especie (edad, sexo, regiones topográficas del animal); 2) de las especies domesticadas en relación al uso económico (carne, lana, transporte); 3) en relación al medio y la derivación en; 4) variabilidad tecnológica.

Por diferentes vías de transmisión los rasgos seleccionados artificialmente sobre la selección natural continua produjeron cambios en segmentos de la población, definiendo: a) formas de transición (en sentido biológico y cultural), b) aparición de otros fenotipos, y c) diferencias dentro de esos nuevos fenotipos. En estas conformaciones actúan las fuentes internas y externas enumeradas.

Para los camélidos no sólo hay dos grupos genéticamente distantes (especies), también la variación se da entre animales dentro de una especie (tipos) y dentro de una población específica.

Entonces, más allá de la carga genética es necesario discriminar cuáles cambios son parte de: a) la normal variación de la población asociada al sexo, edad y regiones corporales por especie; b) las diferentes regiones o zonas de crianza, donde debemos considerar cómo los organismos difieren en sus respuestas a las mismas condiciones, o cómo la respuesta a la misma presión difiere en ambientes diferentes; c) de los mecanismos de selección. Es probable que la llama sobre la cual no ha habido muchos mecanismos de selección tendientes a la especializa-ción, presente una variabilidad mayor que la alpaca, destinada a la producción de fibras, y obviamente que las especies silvestres.

Es importante también a partir de la variabilidad genética determinar bolsones poblacionales y sus diferencias regionales para: 1) evaluar la presencia o no de intercambio de material genético (como otro objeto más de intercambio económico); 2) la divergencia en el progreso genético hueso/fibra.

\section{Propuesta}

Si nuestro objetivo es el estudio del proceso de domesticación debemos ir más allá de la identificación de especies. Brewer (1992) propone pasar de una búsqueda de patrones de rasgos a la identificación de las relaciones entre ellos, e involucra el estudio de los sistemas de subsistencia y la comparación a través del uniformismo y de la analogía de conductas contemporáneas documentadas.

La evidencia arqueológica para las sociedades productoras de alimentos implica el registro en primera instancia de especies domesticadas. En nuestro caso, la domesticación animal sería la existencia de una población de individuos separados fenotípicamente de sus antecesores silvestres, con específicas conformaciones de rebaño acordes con los patrones productivos deseados. En segunda instancia el registro de producción de bienes derivados, consumo y descarte de los mismos.

La evidencia arqueológica ofrece la base empírica pero esta no es una muestra directa de la dinámica de los sistemas socioculturales que le dieron origen (Yacobaccio 1991). Por este motivo las vías propuestas para resolver los problemas planteados son: 1) El reconocimiento empírico de la variabilidad a través del análisis microscópico de fibras de camélidos actuales; 2) El estudio de los factores actuales que reproducen la variación y que regulan la diferente presentación del registro arqueológico; 3) El procesamiento e interpretación de los datos obtenidos a partir del registro arqueológico.

El empleo de la información actual tiende a la búsqueda de los principios que nos permitan interpretar los mecanismos de formación del registro con el cual contamos. Esta proviene de: a) muestras de poblaciones actuales de camélidos con las cuales armamos los patrones comparativos que nos permiten el reconocimiento de la variabilidad en relación a las diferentes fuentes enunciadas, y evaluar diferencias en el progreso genético a partir del cruce de los valores asignados con los que derivan del análisis osteométrico; b) muestras de 
tecnofacturas actuales; c) datos recogidos oralmente con productores de camélidos.

El tema de la variabilidad tecnológica fue sustentado con las fuentes de información b y c (Reigadas 1993). En esta ocasión nos referiremos a la información que proviene del punto a, empleada para evaluar la variabilidad general.

\section{Objetivos}

La necesidad de obtener patrones comparativos responde a múltiples problemas. Por una parte es necesario contar con descripciones lo más ajustadas posibles sobre las poblaciones de camélidos que habitan actualmente las áreas donde efectivizamos los trabajos arqueológicos.

Nuestras designaciones taxonómicas parten del único hecho observable con el que contamos, que es la base empírica que proporcionan nuestras muestras actuales. Estas son empleadas para construir nuestras realidades arqueológicas, a través de la homologación estructural por comparación de rasgos.

En general se observa gran variabilidad, y esto es un hecho fáctico que se debe evaluar a fines de comprender la o las causas que llevan a la diferenciación del registro arqueológico tal cual se presenta en el momento de operar sobre él.

En los casos que estamos evaluando (grosor, color, médula, tipo de fibra) la ampliación de las muestras por sexo, edad para todas las regiones corporales y ambientes diferentes para Lama glama permitirá confirmar o no si las designaciones efectuadas para las muestras arqueológicas de los sitios contemplados que responden eventualmente al patrón doméstico o al tercer agrupamiento (Reigadas 1992, 1993) caen dentro de la normal variación de esta forma doméstica.

En el caso general del estudio del proceso de domesticación animal y en particular del manejo zootécnico, cobra importancia el uso económico de las tropas. Poder ajustar las diferencias morfológicas en relación a los usos es de suma importancia para comprender los incentivos iniciales (obtención de carne, fibra, transporte) que llevaron al hombre a la producción de animales especializados y diferenciados fenotípicamente).
Perkins (1973) hace hincapié sobre los objetivos más importantes del análisis de los restos faunístícos arqueológicos, que serían la determinación de la importancia relativa de los animales ya sean silvestres o domésticos, en relación al tipo de beneficio económico. Lamentablemente en el caso de las fibras, hay mucha pérdida de información (umbrales muy limitados de conservación) que hace muy difícil realizar cuantificaciones paralelas a las osteológicas.

El problema que se plantea es si se puede definir una estructura de rebaño a partir de clases de edad y sexo por el análisis de los vellones, o qué patrón de producción (lanera-carguera) define la estructura visible en el registro. Operativamente es necesario contar con una base de datos que permita evaluar si hay o no soluciones posibles, y en el caso de haberlas qué camino se debe tomar.

La metodología presentada parece ser apropiada para buscar este tipo de información, que concentramos en la determinación de edad, sexo, para cada especie y tipo, con distribución ambiental diferenciada que permita tratar a partir de esta evidencia (fibras) el problema de las diferentes adaptaciones pastoriles en el NOA.

\section{Procedimientos metodológicos}

\section{Variables}

Se reiteran: 1) Las variables elegidas para el análisis morfológico de fibras: color, grosor de la fibra y de la médula, distribución medular por tipo de fibra, tipo de fibra; 2) Los criterios en relación a las observaciones que fueron empleados en el ajuste metodológico y el posterior análisis de los contextos actuales y arqueológicos ya analizados (Reigadas 1991Ms).

Se mantiene la condición de indicadores de primer orden para grosor y características medulares.

\section{Selección de las áreas de estudio}

Se contemplan dos áreas de la Provincia de Jujuy ambientalmente diferenciadas por sus características climáticas y oferta ambiental: 1) Cuenca de Pozuelos y 2) Area de Susques (Cabrera 1957; Ruthsantz y Movia 1975; Castañera 1991). 
A pesar de las diferencias puntuales en los sistemas socioeconómicos actuales (García Fernández y Tecchi 1991), el segmento pastoril juega en ambas roles importantes. Los rebaños entran en el circuito económico familiar y local a partir del uso primario de producción de carne y aprovechamiento del vellón para la producción de fibras textiles y su empleo en manufacturas.

Se observan diferencias en la composición de los rebaños y carga ganadera por hectárea, determinado por el mercado y el ambiente.

En ambas zonas hay pastoreo de llamas cargueras o peladas, intermedias, registrándose en Pozuelos un tercer tipo lanudo. Esta situación nos permite cubrir la posibilidad de obtener una muestra diversificada en cuanto a origen, uso económico y presiones ambientales diferentes.

\section{Cuenca de Pozuelos}

Esta cuenca forma junto a las de Guayatayoc-Salinas Grandes y Olaroz-Cauchan un grupo de tres grandes comarcas endorreicas del altiplano jujeño. La Laguna de Pozuelos (Figura 1) se halla a $22^{\circ} 20^{\prime} \mathrm{S}$ y $66^{\circ} \mathrm{O}$. Ocupa el fondo de un bolsón donde se recogen aguas de una cuenca de $4000 \mathrm{~km}^{2}$.

El clima es frío y seco con características continentales y responde a la zona de la "puna húmeda" (Cabrera 1957), con lluvias estacionales de verano y sequías cíclicas. La vegetación ha sido estudiada e incluida en trabajos regionales de Cabrera (1957, 1968), Ruthsantz y Movia (1975) y García Fernández y Tecchi (1991).

El área perilacustre y márgenes fluviales están cubiertos por un pastizal de Festuca scirpifolia, gramínea de importante valor forrajero. En la época seca en los bordes del piso húmedo de la laguna se desarrolla la "colcha" compuesta por gramíneas rizomatosas.

El área pedemontana está cubierta por tolares de Parastrephia sp. En zonas medanosas aparece la espora Pennisetum chilense, también de importante valor forrajero. Hacia mayores alturas se desarrolla la estepa arbustiva de Fabiana densa y Racharis boliviensis.

Sobre los cerros se observa el desarrollo de vegas de gran extensión.
Las zonas norte y sur presentan una fisonomía diferente. Los agrupamientos de especies vegetales se disponen de acuerdo a los microambientes.

Los pastores tienen las viviendas principales a $3600 \mathrm{~m} . \mathrm{snm}$ en la zona de piedemonte, mientras que los puestos estacionales están en la parte alta de las quebradas y cerca del espejo de agua (Río Grande, Puesto Grande).

La zona de Timón Cruz se encuentra a 4100 m.snm, con formaciones de tolares y vegas de altura, y gran variación en los patrones de asentamiento. Es decir, que la propia heterogeneidad ambiental produce diferencias entre las unidades familiares así como patrones migratorios estacionales.

El acceso a pasturas estables para el ganado está distribuido diferencialmente (García Fernández y Tecchi 1991), al igual que el acceso a cursos de agua.

Las tropas de camélidos consideradas para este trabajo provienen del sector noreste de la cuenca (Río Grande, Puesto Grande) en lo que respecta al tipo lanudo, y del oeste de la cuenca (Timón Cruz) para el tipo carguero o lanudo.

\section{Area de Susques}

Este departamento conforma, junto a los de Yavi, Sta. Catalina, Rinconada, Cochinoca, el altiplano de la Provincia de Jujuy.

La gradiente térmica y pluviométrica genera condiciones más desfavorables hacia el suroeste (Susques) que en el noreste (Pozuelos).

Este ambiente corresponde a la faja de transición de la Puna Seca y Puna Salada.

Este departamento es de menor riqueza ambiental que la cuenca de Pozuelos, por lo que los pastores necesitan acceder a una mayor superficie de pastizales, implicando a su vez mayor trabajo para organizar el pastoreo diario y más grandes distancias que recorrer en el movimiento de los rebaños.

La principal actividad es también la pecuaria con estrategias de hatos múltiples (García Fernández y Tecchi 1991). Los rebaños se trasladan a lo largo del ciclo anual de las vegas y tolares a $3600 \mathrm{~m} . \mathrm{snm}$ 


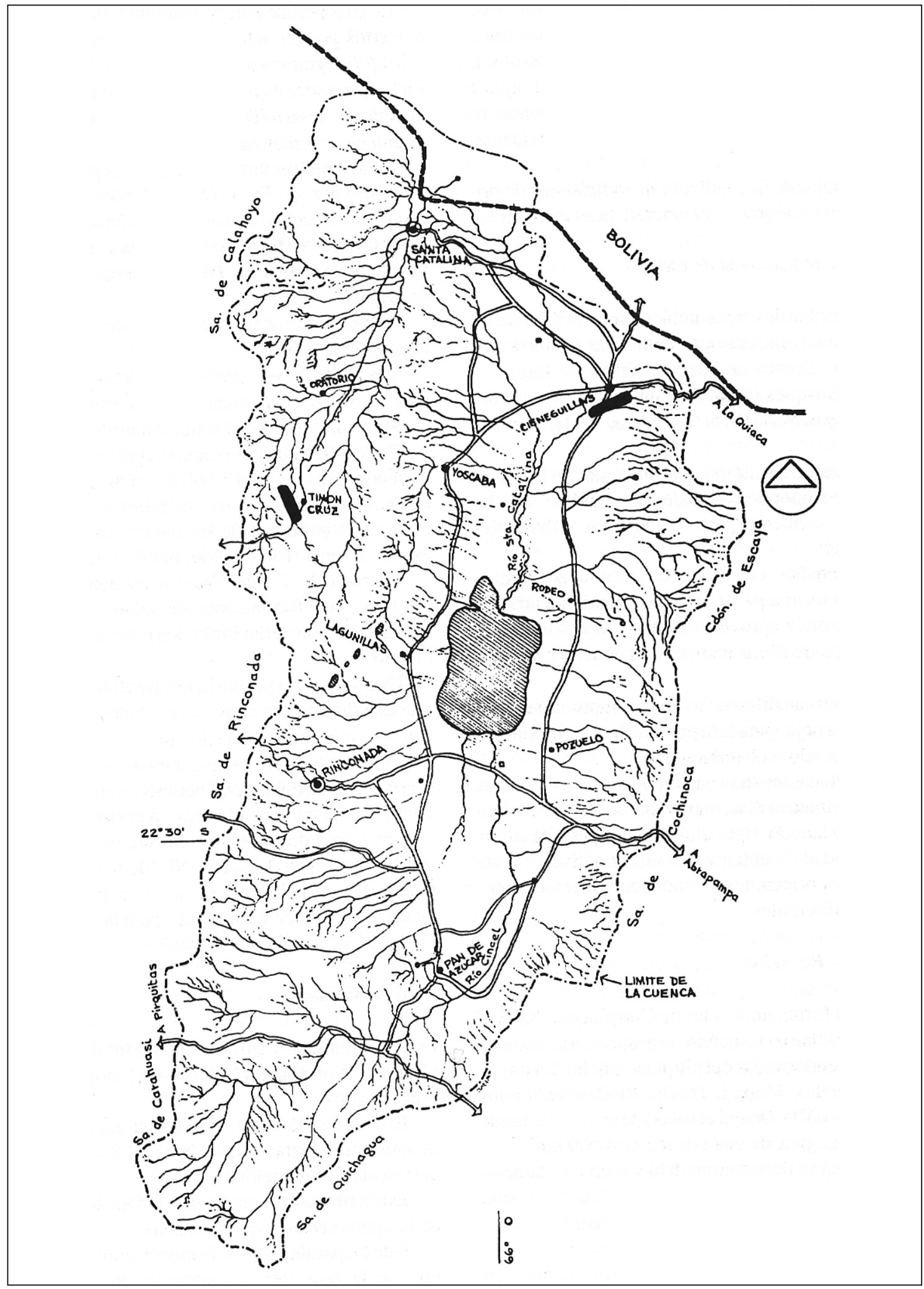

Figura 1. Mapa de la Cuenca de Pozuelos (Tecchi 1990). 


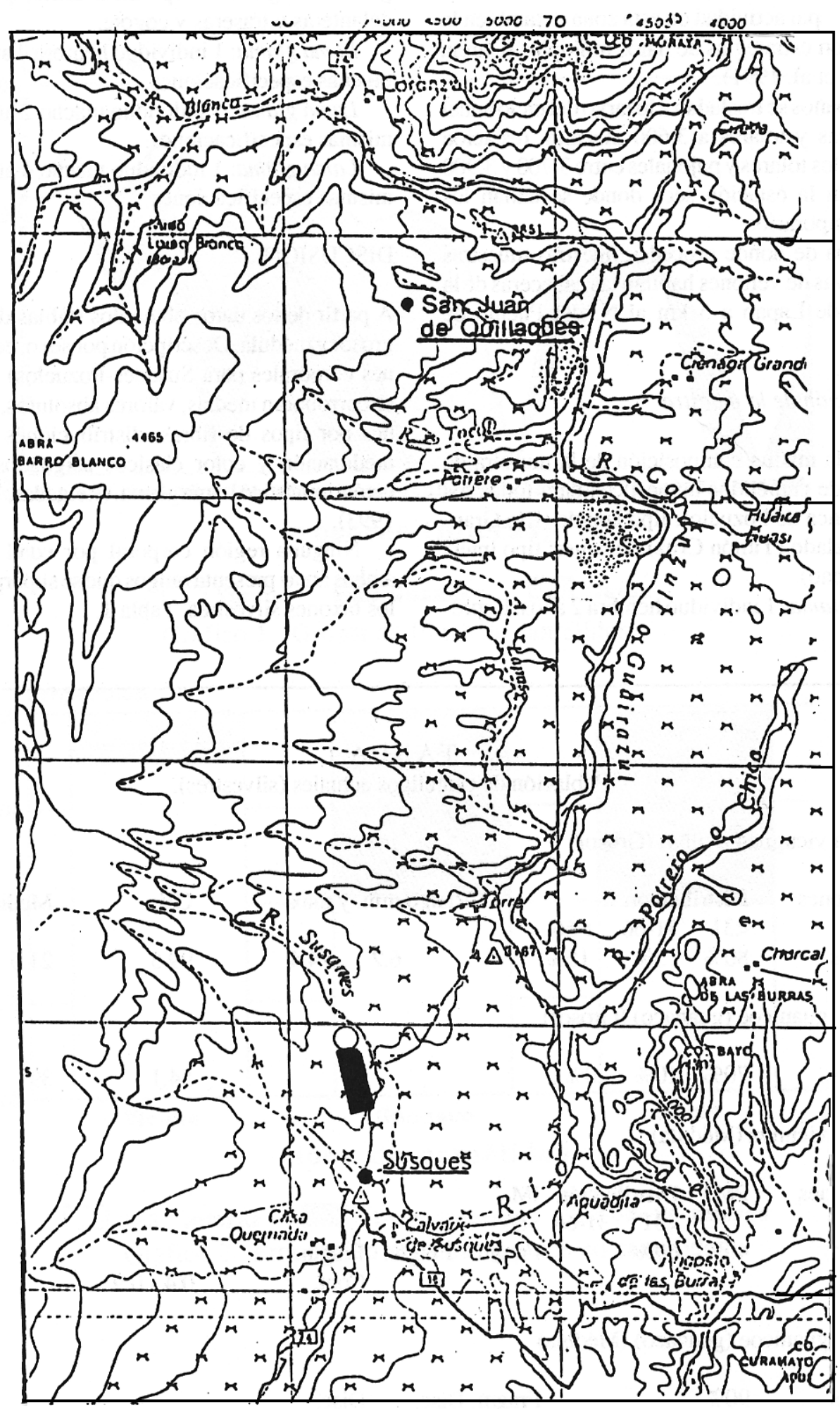

Figura 2. Mapa del área de Susques. 
(estación húmeda) a los tolares y pajonales entre 3700 y 3900 m.snm en la estación seca cuando se usan los puestos temporarios.

La tropa de donde proceden nuestras muestras comparativas de vellones habitan las cabeceras de la quebrada de Lapao $5 \mathrm{~km}$ al norte del pueblo de Susques (Figura 2).

\section{Conformación de la muestra}

Se reitera la misma composición de la muestra de población de camélidos domésticos para todos los casos: Cuenca de Pozuelos: tipo lanudo (Río Grande); tipo pelado (Timón Cruz). Susques: tipo intermedio (Lapao).

Lama glama: Un individuo hembra de dos años, representada por cinco muestras que corresponden: lomo, panza, patas delanteras y traseras y cogote.

Lama glama: Un individuo hembra de cuatro años, con las mismas especificaciones.

Lama glama: Un individuo macho de dos años, con las mismas especificaciones.

Lama glama: Un individuo macho de cuatro años, con las mismas especificaciones.

\section{Discusión}

A partir de los datos obtenidos (Tabla 1, Figura 5, Anexo 1), se corroboran medias, valores absolutos, proporciones por tipos de fibras, distribuciones de grosor, medulación y color clásicos asignados al taxón doméstico Lama glama (Reigadas 1991, 1992, 1993).

Ninguna región corporal por edad y sexo en ambas áreas presenta rangos que se superpongan con los taxones silvestres (Tabla 1).

\section{Pozuelos}

Grosor-regiones topográficas. Se constatan diferencias entre las distintas regiones corporables posibles de cuantificación. Los valores más bajos se registran en lomo y panza. Los más altos en las patas, observando en las traseras los mayores micronajes (en valores absolutos). Las muestras más homogéneas corresponden a la panza.

Grosor-edad-sexo. Se constatan diferencias en relación a la edad en ambos tipos. Los individuos de cuatro años presentan mayores valores.

En relación al sexo en algunos casos los machos presentan los mayores grosores.

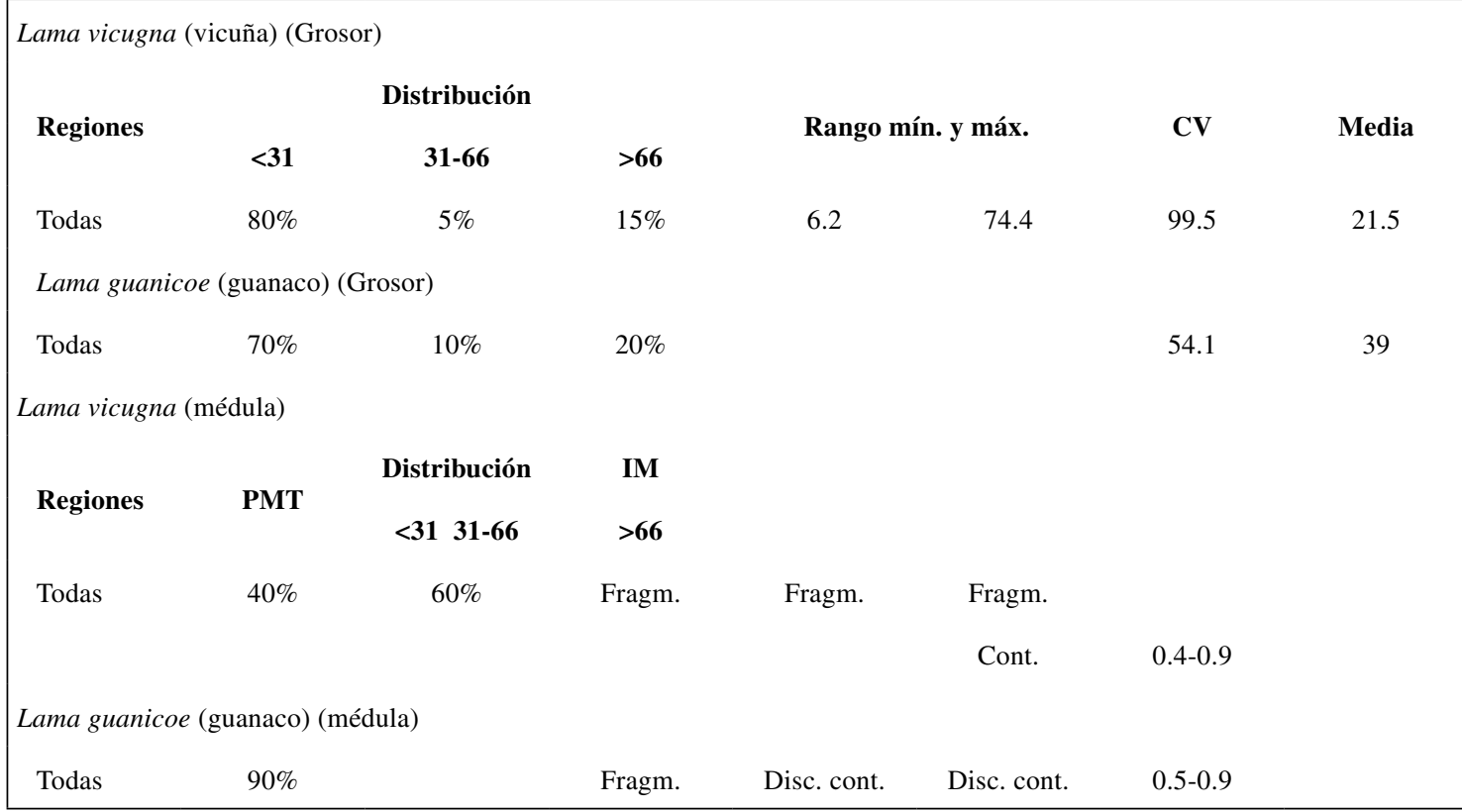

Tabla 1. Población de camélidos actuales (silvestres). 
Entre los tipos se registran rangos de grosor menores en todos los casos para las lanudas.

Médula. El comportamiento de esta variable es típico del taxón doméstico, índices de modulación bajos, con distribución medular en sus tres formas: continua y discontinua en fibras gruesas y medianas y discontinua fragmentada en fibras delgadas. Con registro de fibras secundarias (lanilla) meduladas. Los porcentajes de medulación total más elevados corresponden al tipo carguero y a la región corporal de las patas.

\section{Susques}

Grosor-regiones topográficas. Los valores menores también se dan en el lomo y en la panza. Con presencia de fibras primarias más delgadas. La mayor homogeneidad en torno a valores intermedios se da en la panza que a su vez tiene grosores mayores que el lomo. Las muestras más heterogéneas corresponden a las patas, registrándose los mayores micronajes en las traseras.

Grosor-edad-sexo. Se observaron diferencias en relación a la edad (dos y cuatro años) o primera y segunda esquila. Disminuyendo los valores para cuatro años. Comparando sexos para la misma edad (cuatro años) se verifica incremento de grosor hembra.

Médula. Las características son las propias del taxón como en el caso anterior.

Grosor en relación a las dos áreas. En el caso de Susques no se da la relación esperada por edad (aumento de grosor) y sexo (aumento en machos).

Comparando el tipo carguero e intermedio registramos valores de grosor y medias mayores en el primer tipo.

Todas las muestras correspondientes al tipo lanudo tienen valores absolutos y medias menores que los otros dos tipos (Figura 5).

\section{Caracterización inicial para los diferentes tipos}

En la llama se han identificado dos tipos de animales (Cardozo 1988): kara o pelado y lanudo. Este término tipo es aplicable a los grupos de una especie que difieren en algunas características fenotípicas (Vidal 1967).
Bustinza (1987) registra tres tipos de llama: 1) Peluda (lanuda) o chuco; 2) Pelada o kara (guanaco-llama); 3) Llama intermedia o llama.

Según Laporte y Daza (1980) el tipo lanudo está especializado en producción de fibra y carne, mientras que el pelado en carne y carga.

En nuestro caso para muestras comparativas de Susques estaríamos descartando el fenotipo lanudo en primer término por una cuestión visual. Estas presentan mechones de fibras en las orejas y cabeza, y tienen mayor cobertura de vellón en la mitad superior del cuello, características que no se presentan en la tropa de procedencia de nuestras muestras.

Por otra parte, los porcentajes de medulación que registramos son superiores a los de este tipo que sólo alcanza 35\% según Vidal (1967) y Maquera Llano (1991). Esta característica separa también a nuestro tipo lanero de Pozuelos de este lanudo.

Respecto al diámetro y la media, en los tipos intermedios y pelados son superiores los correspondientes a lanudos con rangos que van entre 10 y $150 \mu$ (micras) (Vidal 1967). Según nuestro análisis las medias en casi todos los casos superan el $25 \%$ que da este autor para lanuda.

Visualmente las intermedias presentan menor cobertura que las lanudas, pero mayor que en las peladas, donde a su vez se registra gran cantidad de fibras primarias (pelos de mayor grosor). Nuestras medias para las muestras de Susques se acercan a las de tipo intermedio sin presencia de pelos muy gruesos, con una morfología externa acorde con el tipo.

Concluimos a partir de esta primera aproximación que en el área de Susques descartamos el tipo lanero peruano y el lanudo registrado en Pozuelos. Las muestras provienen de un segmento de la población que definimos como intermedio, observándose la presencia del tipo pelado, que será incluido en las muestras comparativas en el futuro.

En Pozuelos hasta el momento se designaron los tipos pelado y lanudo. También en esta área se contempla la ampliación de la muestra habiéndose observado tropas correspondientes a los tres tipos en Timón Cruz. 


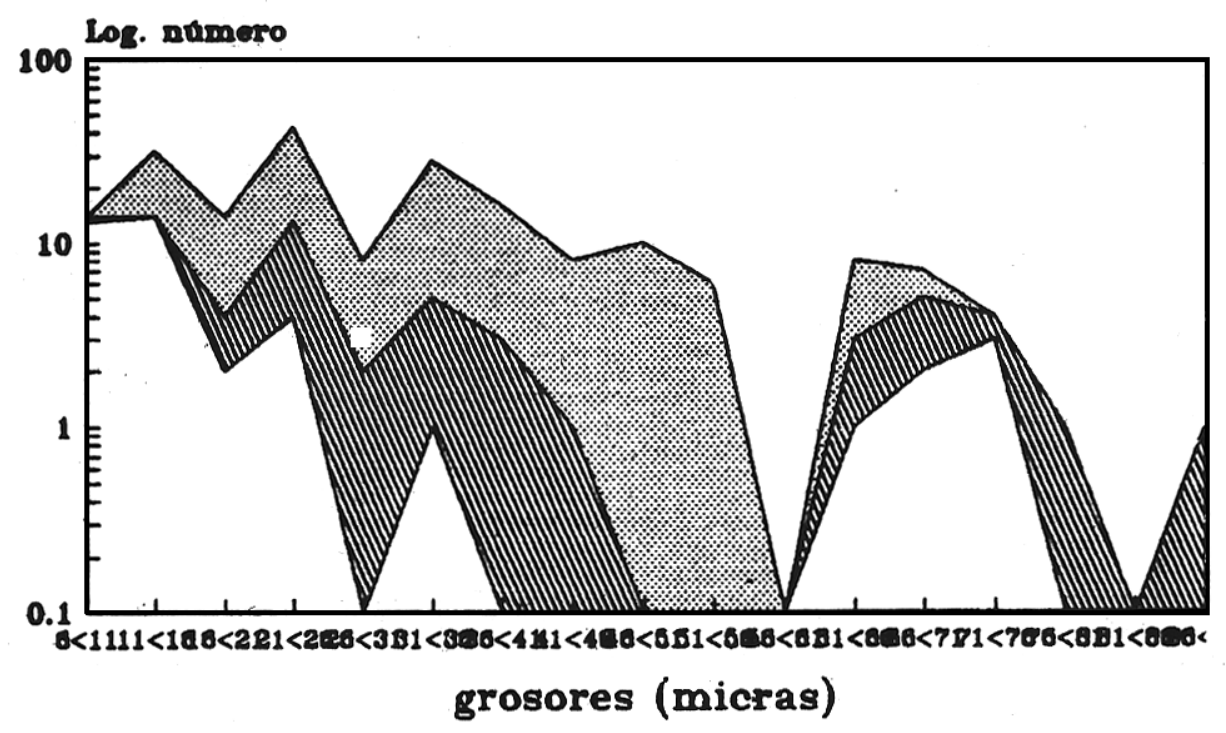

$\square$ Vicuna AlliV Guanaco uama

Figura 3. Grosor de fibras de camélidos.

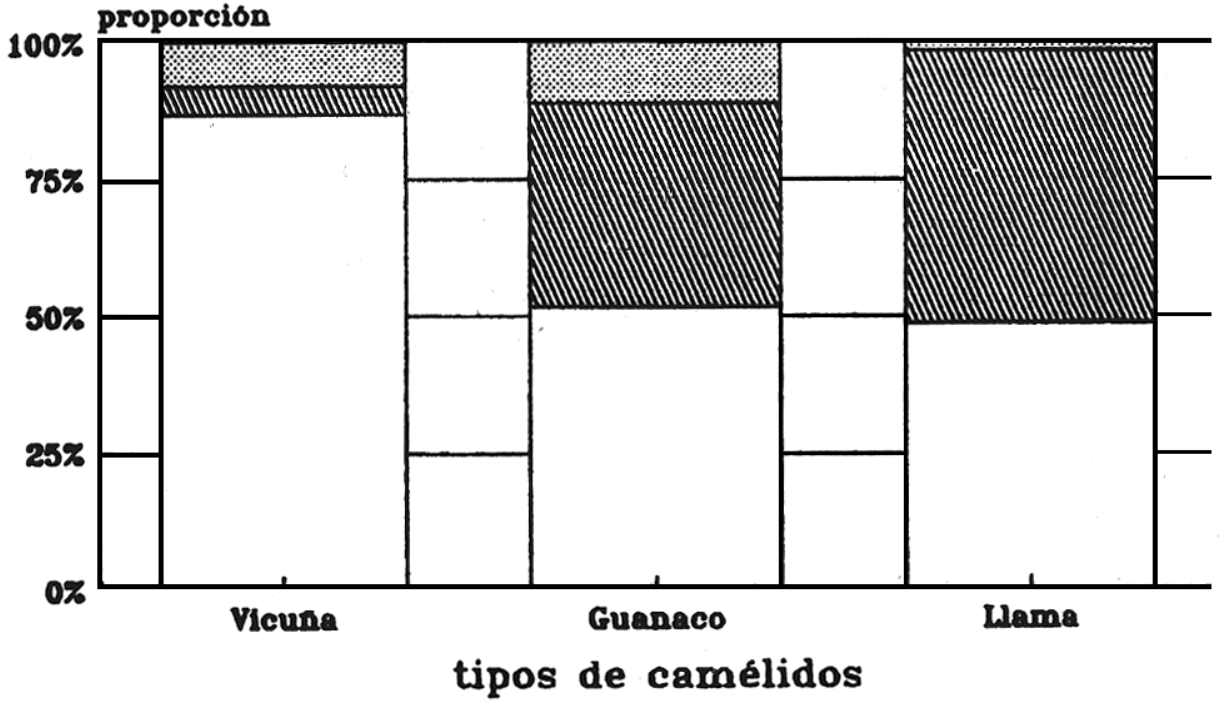

0-31 micras 31-66 micras 66-76 micras

Figura 4. Proporción de fibras y pelos en camélidos. 


\section{Conclusión}

El empleo de los datos actuales revelados son de utilidad para:

1) Armar muestras como patrones comparativos.

2) Evaluar a partir de descripciones morfológicas más detalladas las asignaciones realizadas en las muestras arqueológicas. Ya vimos que hemos descartado la posible superposición de rangos asumidos para formas domésticas con formas silvestres.

3) Conocer la variabilidad actual como base de inferencias para la interpretación de la variabilidad del registro arqueológico. Esto en relación a la posibilidad planteada de reconocer patrones productivos (carne, lana, transporte) a partir de la composición diferencial de los vellones. Por el momento las variables seleccionadas y las cuantificaciones realizadas permiten la diferenciación de tipos a partir del análisis microscópico de fibras.

4) Proponer hipótesis sobre los incentivos (plasmados en patrones de producción) que pueden haber funcionado como movilizadores del proceso de domesticación.

5) Proponer explicaciones acerca de las técnicas de producción de fibras para la elaboración de manufacturas textiles y la variabilidad observada en las mismas.

En relación a los dos grupos de preguntas ya enunciadas y que dieron origen a este trabajo, el carácter metodológico de las primeras y explicativo de las segundas a su vez se ven cruzadas por los tres ejes de trabajo propuestos al comenzar el empleo de esta vía de análisis para detectar y explicar el proceso de domesticación (Reigadas 1992).

Para el problema taxonómico la información presentada hizo posible definir morfológicamente a partir de las variables propuestas los agrupamientos correspondientes a las poblaciones actuales de camélidos silvestres y domésticos, posibilitando la adscripción de las muestras arqueológicas a los taxones estudiados (Anexo 1).

Se ha podido comenzar a evaluar los efectos que las diferentes fuentes de variación ejercen en los rangos asumidos.
Se registraron variaciones por edad, sexo y regiones corporales que han posibilitado el control de las asignaciones, constatándose que ningún rango asignado a forma doméstica o tercer agrupamiento se superpone con formas silvestres, sino que están incluidas en las descripciones aquí realizadas.

Vemos que este tipo de información ha aportado más a la resolución metodológica.

Se observaron también diferencias en la composición de los pelajes que en la actualidad responden a usos económicos diferentes y son posibles de cuantificación. Siendo la determinación de tipos válido para el problema de patrones ganaderos productivos.

La información obtenida se incorpora en un marco más explicativo.

Con respecto a la última fuente de variación, el factor ambiental, se registra en Susques una situación anómala en cuanto a la disminución de los grosores con la edad. Las variaciones de rangos de grosor están en relación con las pasturas disponibles, o el acceso a los diferentes recursos que requiere el animal (pasturas-agua).

En esta zona se registran diferentes movimientos que deben efectuar las tropas en relación a la disponibilidad espacial de los recursos y diferencias cualitativas en las comunidades vegetales con respecto al área de Pozuelos.

Esta anomalía puede ser provocada por estrés en la captura de recursos por parte de las poblaciones descritas. Esta situación puede tomarse en cuenta en casos arqueológicos donde la curva de incremento de grosor se invierta.

En relación al problema evolutivo consideramos las diferencias en las poblaciones de la cuenca de Pozuelos. En las fibras del grupo lanero se han producido variaciones a partir de cruza con alpaca. Los rangos de grosor se asemejan a los de este taxón (Calle Escobar 1984). Se conformó un tipo específico que corresponde osteológicamente al morfotipo llama y por cobertura alpaca. Se constata un cambio evolutivo, donde la variación fenotípica aparece primero en las fibras.

Esta situación permitirá evaluar en el registro arqueológico la aparición temprana de variación 


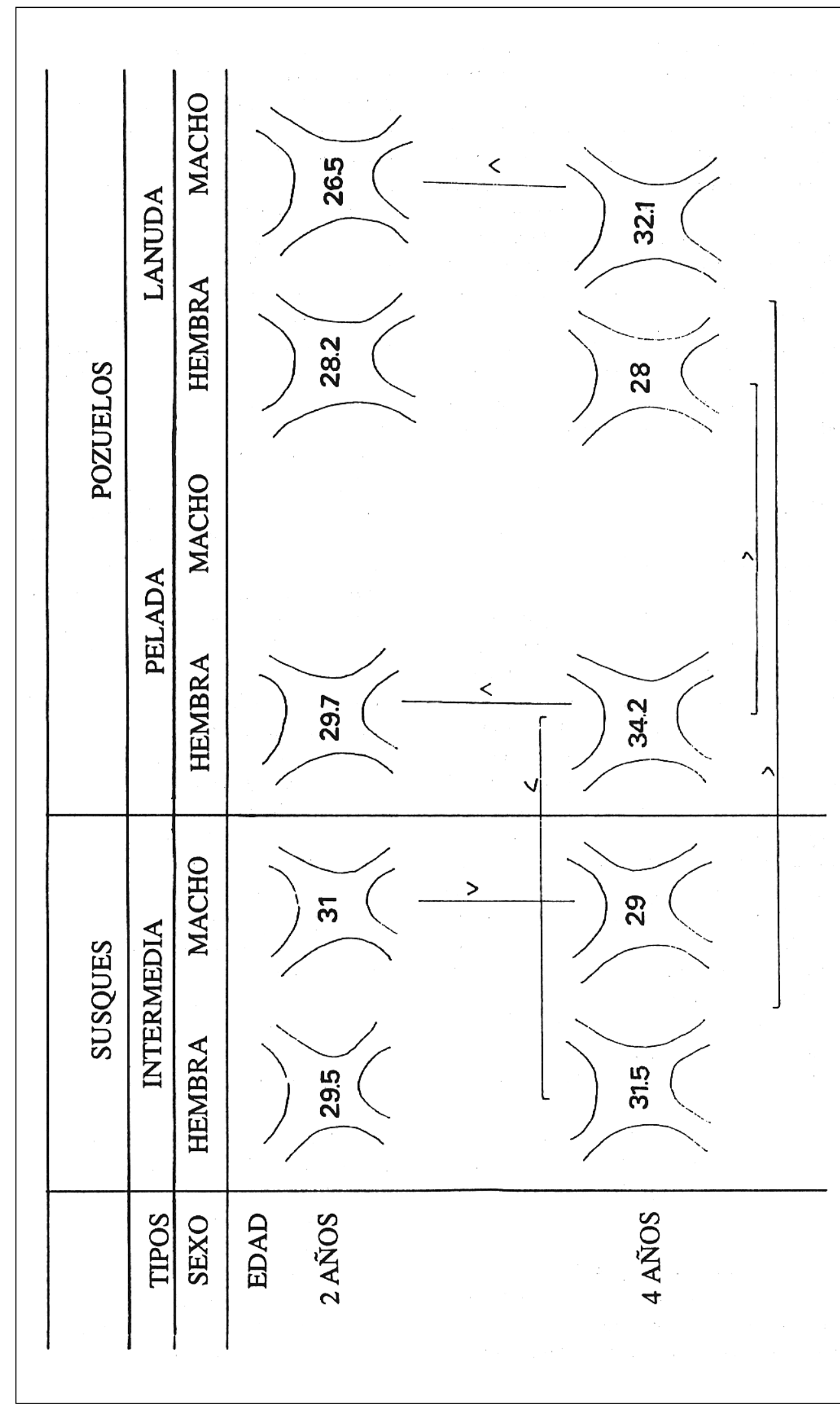

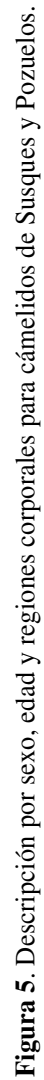


en los vellones (tercer agrupamiento) como caso análogo.

La descripción más detallada de estas poblaciones es de importancia también para el problema tecnológico, pudiendo a partir de ella relacionar el tipo de manufacturas y empleo de las mismas por la determinación de la región corporal empleada y la selección diferencial de fibras.

Conocer la selección ejecutada en cada caso permite a su vez controlar las designaciones taxonómicas efectuadas a partir de manufacturas.

La información presentada ayudará a contrastar las hipótesis formuladas previamente.

Para la H1: "Las diferentes fuentes de variabilidad van a influir diferencialmente en los silvestres y domésticos", tenemos la información relativa a formas domésticas.

Los datos de formas silvestres empleados corresponde a las muestras de Lama guanicoe y Lama vicugna utilizados hasta el momento como muestras comparativas.

Para la H2: "En formas domésticas la variabilidad por uso económico va a tener preeminencia sobre las fuentes ambientales", los datos que corresponden a las diferencias de los tres mantos corroborarían la formulación.

Faltarían análisis de densidad de fibras primarias y secundarias para poder tener otro elemento más diferenciador a nivel arqueológico entre las cargueras e intermedias (productoras de carne).

Consideremos ampliar la muestra en Susques a fines de verificar si la anomalía se reitera. Así podríamos evaluar la posibilidad de diferencias en la alimentación y costo de traslado para el rebaño.

Para la H3: "En los animales domésticos se va a registrar mayor variabilidad que en los silvestres", debemos como en la H1 obtener la información ampliada para taxones silvestres.

\section{Nuevas perspectivas para la explicación del proceso de domesticación}

Comparando la muestra total de muestras arqueológicas de los sitios de procedencia (Tabla 2) se ha llegado a las siguientes conclusiones:
1) El "tercer grupo" se aleja estructuralmente de los patrones silvestres.

2) El "tercer grupo" corresponde morfológicamente al patrón doméstico actual.

3) La forma doméstica designada mantiene la estructura del "tercer grupo".

4) El "tercer grupo" concuerda con el tipo que definimos en este trabajo como llama Intermedia.

Esta población ("tercer grupo") separada de las dos variantes silvestres hace aproximadamente 9000 años como registramos en Inca Cueva, Cueva 4, capa 2 y Quebrada Seca 3, niveles inferiores (Reigadas 1992, 1994), respondería al proceso de especiación a partir de selección natural propuesta como primer paso de generación de variación.

Un segundo paso de selección a nivel de fenotipo introduce el control humano posibilitando por un lado la conservación fenotípica del grupo y el posterior control reproductivo.

Las muestras arqueológicas asignadas a formas domésticas y con contexto asociado a una situación de pastoreo, por ejemplo la capa $\mathrm{D}$ de Huachichocana Cueva III, reflejarían esta situación (Reigadas 1992).

Para concluir y como hipótesis general para futuros trabajos consideramos que la selección cultural opera sobre un tipo generalizado de camélido semejante al tipo intermedio definido en este trabajo, que promueve a posteriori un patrón productivo especializado en la obtención de carne y fibra.

Consideramos también que si el progreso genético diferencial hueso/fibra ubica al vellón como indicador temprano de variación morfológica, la correlación con indicadores osteométricos que muestran contemporaneidad de elementos óseos adscriptos a formas silvestres (Elkin et al. 1992) y vellones asignados a formas silvestres y al tercer grupo (Reigadas 1995), en momentos tempranos, correspondería a la primera etapa de este proceso que surge en un contexto de caza y recolección.

La correlación entre ambos indicadores, en momentos más tardíos con contexto de pastoreo, es coincidente a formas domésticas tanto en 


\begin{tabular}{|c|c|c|c|c|c|c|c|c|c|c|}
\hline ICC4 & $\mathbf{C}$ & ICA1 & $\mathrm{C}$ & ICCV & $\mathbf{C}$ & CHCIII C & $\mathrm{CHCV}$ & $\mathbf{C}$ & QS3 & CN \\
\hline \multicolumn{11}{|c|}{ PRECERAMICO TEMPRANO } \\
\hline & & & & & & & & & Inf. & \\
\hline$*$ & $* * 2$ & & & & & $* * * \mathrm{E} 3$ & & & 0 & $2 \mathrm{~b} 19$ \\
\hline \multirow[t]{11}{*}{0} & $?$ & & & & & & & & $* *$ & $2 \mathrm{~b} 18$ \\
\hline & & & & & & & & & $* *$ & $2 \mathrm{~b} 16$ \\
\hline & & & & & & & & & $* *$ & $2 \mathrm{~b} 14$ \\
\hline & & & & & & & & & $0^{* *}$ & $2 \mathrm{~b} 13$ \\
\hline & & & & & & & & & $0 * *$ & $2 \mathrm{~b} 12$ \\
\hline & & & & & & & & & Int. & \\
\hline & & & & & & & & & $0 * *$ & $2 \mathrm{~b} 11$ \\
\hline & & & & & & & & & $0 * *$ & $2 \mathrm{~b} 10$ \\
\hline & & & & & & & & & $0 * *$ & $2 \mathrm{~b} 9$ \\
\hline & & & & & & & & & $0 * *$ & $2 \mathrm{~b} 7 / 6$ \\
\hline & & & & & & & & & $7 * 0 * *$ & $2 \mathrm{~b} 6$ \\
\hline \multicolumn{11}{|c|}{ PRECERAMICO TARDIO } \\
\hline & & & & & & & & & Sup. & \\
\hline$* * *$ & $1 \mathrm{a}$ & & & & & ? E2 & & & $* 0 * *$ & $2 \mathrm{~b} 5$ \\
\hline \multirow[t]{3}{*}{$? 0$} & & & & & & & & & $* 0 * *$ & $2 \mathrm{~b} 4$ \\
\hline & & & & & & & & & $0 * *$ & $2 \mathrm{~b} 3$ \\
\hline & & & & & & & & & $0 * *$ & $2 \mathrm{~b} 2$ \\
\hline \multicolumn{11}{|c|}{ AGROALFARERO } \\
\hline & & $0+? 1$ & & & & $0 * * \mathrm{El}$ & & & & \\
\hline & & & & & & $?$ & & & & \\
\hline & & $* *+2 *$ & & & & $* * * \mathrm{D}$ & & & & \\
\hline & & & & & & + & & & & \\
\hline & & & & & & $* * * \mathrm{C}$ & $+\mathrm{B}$ & & & \\
\hline & & & & & & + & & & & \\
\hline \multicolumn{3}{|c|}{ POSTHISPANICO } & & & & & $1-*$ & guanaco & & \\
\hline & & & & & & $+\mathrm{B}$ & $2-* *$ & vicuña & & \\
\hline & & & & & & & 3- 0 & tercer grupo & & \\
\hline & & & & & & & $4-+$ & llama & & \\
\hline & & & & & & & $5-7$ & sin design. & & \\
\hline
\end{tabular}

Tabla 2. Cuadro de taxones identificados en el NOA a partir del análisis de fibras.

elementos óseos como vellones; como resultado de la evolución de esta población diferenciada que persiste gracias al control humano como respuesta ante algún beneficio económico (carne-fibra), se consolida a través del control reproductivo, definiendo este producto doméstico en términos biológicos y culturales con un valor específico de uso.
La metodología propuesta nos ha permitido observar la variabilidad a partir del análisis de los vellones, y comenzar a armar la base de datos apropiada para contemplar el problema de la transmisión de alguna variabilidad (especiación) y su representación diferencial (tipos), para poder estudiar los cambios, producto de la evolución cultural y la diversidad resultante específicamente en el proceso de domesticación animal. 


\section{ANEXO 1 DATOS OBTENIDOS}

Descripción por sexo, edad y regiones corporales para Susques-Pozuelos. Población de camélidos actuales (domésticos).

\begin{tabular}{|c|c|c|c|c|c|c|c|}
\hline \multicolumn{8}{|c|}{$\begin{array}{l}\text { Cuenca de Pozuelos } \\
\text { Cepa carguera (Timón Cruz) } \\
\text { Hembra } 2 \text { años (Grosor) }\end{array}$} \\
\hline \multirow{2}{*}{ Regiones } & \multicolumn{3}{|c|}{ Distribución } & \multirow{2}{*}{\multicolumn{2}{|c|}{ Mín. y Máx. }} & \multirow{2}{*}{ CV (\%) } & \multirow{2}{*}{ Media } \\
\hline & $<31$ & $31-66$ & $>66$ & & & & \\
\hline Todas & $60 \%$ & $40 \%$ & & 14.2 & 53.5 & & 29.7 \\
\hline Lomo & $60 \%$ & $40 \%$ & & & 39.2 & & 24.5 \\
\hline Panza & $70 \%$ & $30 \%$ & & 16 & 53.5 & & 29.2 \\
\hline Pata d. & $30 \%$. & $70 \%$ & & 21.4 & & 14 & \\
\hline Pata t. & $40 \%$ & $60 \%$ & & 21.4 & 50 & 29 & 35.5 \\
\hline Cogote & $70 \%$ & $30 \%$ & & 16 & 46.6 & 41 & 27.8 \\
\hline \multicolumn{8}{|c|}{ Hembra 4 años (Grosor) } \\
\hline Todas & $60 \%$ & $33 \%$ & 7 & 10.7 & 92.8 & 61.3 & 34.2 \\
\hline Lomo & $70 \%$ & & $30 \%$ & 10.7 & 90 & 89.4 & 37 \\
\hline Panza & $60 \%$ & $40 \%$ & & 12.5 & 64.2 & & 36.5 \\
\hline Pata d. & $60 \%$ & $40 \%$ & & 16 & 39.2 & 29 & 27.4 \\
\hline Pata t. & $50 \%$ & $50 \%$ & & 12.6 & 64.2 & 51 & 34.2 \\
\hline Cogote & $50 \%$ & $40 \%$ & 10 & 17.8 & 71.4 & & 35.3 \\
\hline
\end{tabular}

\begin{tabular}{|c|c|c|c|c|c|}
\hline \multicolumn{6}{|c|}{$\begin{array}{l}\text { Cepa carguera (Timón Cruz) } \\
\text { Hembra } 2 \text { años (Médula) }\end{array}$} \\
\hline \multirow{2}{*}{ Regiones } & \multirow{2}{*}{ PMT } & \multicolumn{3}{|c|}{ Distribución } & \multirow{2}{*}{ IM } \\
\hline & & $<31$ & $31-66$ & $>66$ & \\
\hline Lomo & $50 \%$ & & $\begin{array}{l}\text { Cont. } \\
\text { Disc. }\end{array}$ & & $0.2-0.3$ \\
\hline Panza & $70 \%$ & $\begin{array}{l}\text { Frag. } \\
\text { Disc. }\end{array}$ & Cont. & & $0.2-0.5$ \\
\hline Pata d. & $90 \%$ & Frag. & $\begin{array}{l}\text { Cont. } \\
\text { Disc. }\end{array}$ & & 0.2 \\
\hline Pata t. & $60 \%$ & & $\begin{array}{c}\text { Fragm. } \\
\text { Disc. } \\
\text { Cont. }\end{array}$ & & $0.1-0.3$ \\
\hline Cogote & $80 \%$ & Frag. & $\begin{array}{l}\text { Cont. } \\
\text { Disc. }\end{array}$ & & $0.3-0.6$ \\
\hline \multicolumn{6}{|c|}{ Hembra 4 años (Médula) } \\
\hline Lomo & $70 \%$ & Frag. & $\begin{array}{l}\text { Cont. } \\
\text { Disc. }\end{array}$ & Cont. & $0.2-0.7$ \\
\hline Panza & $70 \%$ & Frag. & Cont. & & $0.3-0.6$ \\
\hline Pata d. & $80 \%$ & & Cont. & & $0.1-0.3$ \\
\hline Pata t. & $70 \%$ & Fragm. & Cont. & & $0.2-0.5$ \\
\hline Cogote & $70 \%$ & Cont. & Cont. & & $0.3-0.6$ \\
\hline
\end{tabular}




\begin{tabular}{|c|c|c|c|c|c|c|}
\hline \multicolumn{7}{|c|}{$\begin{array}{l}\text { Cepa Lanera (Río Grande) } \\
\text { Macho } 2 \text { años (Grosor) }\end{array}$} \\
\hline \multirow{3}{*}{$\begin{array}{l}\text { Regiones } \\
\text { Todas }\end{array}$} & \multicolumn{2}{|c|}{ Distribución } & \multirow{2}{*}{\multicolumn{2}{|c|}{ Mín. y Máx. }} & \multirow{2}{*}{$\mathrm{CV}(\%)$} & \multirow{2}{*}{ Media } \\
\hline & $<31$ & $31-66>66$ & & & & \\
\hline & $75 \%$ & $25 \%$ & 7.1 & 46.4 & & 26.5 \\
\hline Lomo & $70 \%$ & $30 \%$ & 16 & 46.4 & & 28.1 \\
\hline Panza & $80 \%$ & $20 \%$ & 14.2 & 39.2 & & 23.5 \\
\hline Pata d. & $60 \%$ & $40 \%$ & 16 & 39.2 & & 26.9 \\
\hline Pata t. & $80 \%$ & $20 \%$ & 7.1 & 39.2 & & 26 \\
\hline Cogote & $80 \%$ & $20 \%$ & 14.2 & 42.8 & & 27 \\
\hline \multicolumn{7}{|c|}{ Macho 4 años (Grosor) } \\
\hline Todas & $60 \%$ & $40 \%$ & 14.2 & 58.9 & & 32.1 \\
\hline Lomo & $60 \%$ & $40 \%$ & 16 & 44.6 & 29.7 & 30.3 \\
\hline Panza & $50 \%$ & $50 \%$ & 17.8 & 58.9 & & 34.9 \\
\hline Pata d. & $50 \%$ & $50 \%$ & 14.2 & 51.7 & & 33.2 \\
\hline Pata t. & $50 \%$ & $50 \%$ & 21.4 & 42.8 & & 32.5 \\
\hline Cogote & $50 \%$ & $50 \%$ & 14.2 & 44.6 & 43.8 & 29.4 \\
\hline \multicolumn{7}{|c|}{ Hembra 2 años (Grosor) } \\
\hline Todas & $70 \%$ & $30 \%$ & 13.2 & 57.1 & & 28.2 \\
\hline Lomo & $90 \%$ & $10 \%$ & 14.2 & 50 & & 25.8 \\
\hline Panza & $80 \%$ & $20 \%$ & 14.2 & 35.7 & 29 & 25.8 \\
\hline Pata d. & $20 \%$ & $80 \%$ & 17.8 & 57.1 & & 39.6 \\
\hline Pata t. & $90 \%$ & $10 \%$ & 17.8 & 46.4 & & 26.1 \\
\hline Cogote & $90 \%$ & $10 \%$ & 13.2 & 35.7 & & 24.3 \\
\hline \multicolumn{7}{|c|}{ Hembra 4 años (Grosor) } \\
\hline Todas & $80 \%$ & $20 \%$ & 12.5 & 50 & & 25.4 \\
\hline Lomo & $60 \%$ & $40 \%$ & 14.2 & 50 & & 26.4 \\
\hline Panza & $70 \%$ & $30 \%$ & 19.6 & 39 & & 26.4 \\
\hline Pata d. & $70 \%$ & $30 \%$ & 21.4 & 50 & & 29.6 \\
\hline Pata t. & $100 \%$ & & 12.5 & 32 & & 22.2 \\
\hline
\end{tabular}


INCIDENCIA DE LOS FACTORES DE VARIACION EN LAS ESPECIES DE CAMELIDOS Y TIPOS DOMESTICOS..

\begin{tabular}{|c|c|c|c|c|c|}
\hline \multicolumn{6}{|c|}{$\begin{array}{l}\text { Cepa Lanera (Río Grande) } \\
\text { Macho } 2 \text { años (Médula) } \\
\text { Corteza oscura. No pudo registrarse }\end{array}$} \\
\hline \multicolumn{6}{|c|}{ Macho 4 años (Médula) } \\
\hline \multirow{2}{*}{ Regiones } & \multirow{2}{*}{ PMT } & \multicolumn{3}{|c|}{ Distribución } & \multirow{2}{*}{ IM } \\
\hline & & $<31$ & $31-66$ & $>66$ & \\
\hline Lomo & $80 \%$ & Frag. & $\begin{array}{l}\text { Disc. } \\
\text { Cont. }\end{array}$ & & $0.2-0.3$ \\
\hline Panza & $50 \%$ & Frag. & Cont. & & $0.1-0.4$ \\
\hline Pata d. & Corteza oscura & & & & \\
\hline Pata t. & Corteza oscura & & & & \\
\hline Cogote & $40 \%$ & $\begin{array}{l}\text { Cont. } \\
\text { Disc. }\end{array}$ & & & $0.1-0.2$ \\
\hline \multicolumn{6}{|c|}{ Hembra 2 años (Médula) } \\
\hline Lomo & $70 \%$ & $\begin{array}{l}\text { Fragm. } \\
\text { Cont. }\end{array}$ & $\begin{array}{c}\text { Fragm. } \\
\text { Cont. }\end{array}$ & & $0.1-0.4$ \\
\hline Panza & $70 \%$ & $\begin{array}{c}\text { Fragm. } \\
\text { Cont. }\end{array}$ & $\begin{array}{c}\text { Fragm. } \\
\text { Cont. }\end{array}$ & & $0.2-0.5$ \\
\hline Pata d. & $90 \%$ & $\begin{array}{c}\text { Fragm. } \\
\text { Disc. }\end{array}$ & Cont. & & $0.1-0.5$ \\
\hline Pata t. & $50 \%$ & Cont. & Cont. & & $0.3-0.4$ \\
\hline Cogote & & $\begin{array}{c}\text { Fragm. } \\
\text { Cont. }\end{array}$ & $\begin{array}{c}\text { Fragm. } \\
\text { Cont. }\end{array}$ & & 0.2 \\
\hline \multicolumn{6}{|c|}{ Hembra 4 años (Médula) } \\
\hline Lomo & $50 \%$ & & Cont. & & $0.2-0.4$ \\
\hline Panza & $60 \%$ & Fragm. & $\begin{array}{l}\text { Fragm. } \\
\text { Disc. } \\
\text { Cont. }\end{array}$ & & $0.2-0.4$ \\
\hline Pata d. & $70 \%$ & Fragm. & $\begin{array}{l}\text { Cont. } \\
\text { Disc. }\end{array}$ & & $0.2-0.5$ \\
\hline Pata t. & $50 \%$ & Fragm. & $\begin{array}{l}\text { Cont. } \\
\text { Disc. }\end{array}$ & & $0.2-0.4$ \\
\hline Cogote & $60 \%$ & & $\begin{array}{l}\text { Cont. } \\
\text { Disc. }\end{array}$ & & $0.2-0.4$ \\
\hline
\end{tabular}


Susques

Intermedia (Qda. de Lapao)

Macho 2 años (Grosor)

\begin{tabular}{|c|c|c|c|c|c|c|c|}
\hline \multirow{2}{*}{ Regiones } & \multicolumn{3}{|c|}{ Distribución } & \multirow{2}{*}{\multicolumn{2}{|c|}{ Rango Mín. y Máx. }} & \multirow{2}{*}{ CV $(\%)$} & \multirow{2}{*}{ Media } \\
\hline & $<31$ & $31-66$ & $>66$ & & & & \\
\hline Todas & $57 \%$ & $43 \%$ & & 13 & 64.2 & & 31 \\
\hline Lomo & $60 \%$ & $40 \%$ & & 13.2 & 57.1 & 50 & 30 \\
\hline Panza & $20 \%$ & $80 \%$ & & 28.5 & 64.2 & 29 & 41.7 \\
\hline Pata d. & $70 \%$ & $30 \%$ & & 14.2 & 50 & 47.9 & 27.6 \\
\hline Pata t. & $70 \%$ & $30 \%$ & & 13.2 & & 62.5 & \\
\hline
\end{tabular}

Macho 4 años (Grosor)

\begin{tabular}{|l|l|l|l|lc|r|r|}
\hline Todas & $60 \%$ & $40 \%$ & & 10,7 & 57.1 & & 29 \\
\hline Lomo & $70 \%$ & $30 \%$ & & 10.7 & 41 & & 23.7 \\
\hline Panza & $30 \%$ & $70 \%$ & & 28.5 & 42.8 & 16.8 & 35.7 \\
\hline Pata d. & $70 \%$ & $30 \%$ & & 10.7 & 50 & 50.3 & 24.9 \\
\hline Pata t. & $80 \%$ & $20 \%$ & & 27.3 & 57.1 & & 29.1 \\
\hline Cogote & $50 \%$ & $50 \%$ & & 14.2 & 57.1 & & 33.4 \\
\hline
\end{tabular}

Hembra 4 años (Grosor)

\begin{tabular}{|l|l|l|l|ll|l|l|}
\hline Todas & $67 \%$ & $28 \%$ & $5 \%$ & 14.2 & 78.5 & & 31,5 \\
\hline Lomo & $60 \%$ & $40 \%$ & & 16 & 62.5 & & 32,8 \\
\hline Panza & $70 \%$ & $30 \%$ & & 14.2 & 42.8 & 34 & 26,9 \\
\hline Pata d. & $60 \%$ & $40 \%$ & & 17.8 & 60.7 & 46 & 32,2 \\
\hline Pata t. & $80 \%$ & & $20 \%$ & 17.8 & 78.5 & 62 & 34,7 \\
\hline
\end{tabular}

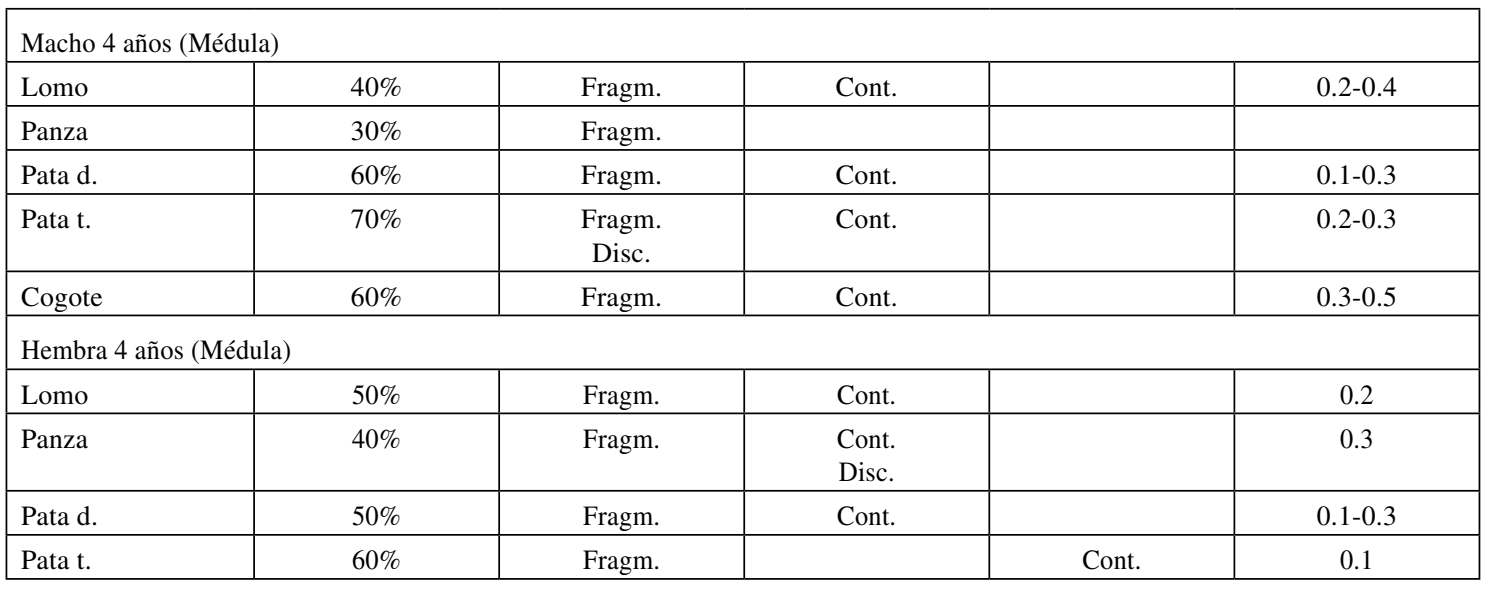


INCIDENCIA DE LOS FACTORES DE VARIACION EN LAS ESPECIES DE CAMELIDOS Y TIPOS DOMESTICOS...

UNIDADES DE ANALISIS

\begin{tabular}{|c|c|c|c|c|c|}
\hline Sitio & Capa & Altitud & Ubicación temporal & Fechas & Muestras \\
\hline ICC4 & $\begin{array}{c}2 \\
1 \mathrm{a}\end{array}$ & $\begin{array}{l}3650 \\
\text { Prep. }\end{array}$ & $\begin{array}{c}\text { Precerámico Temprano } \\
\text { Precerámico Tardío }\end{array}$ & $\begin{array}{l}10620 \pm 100 \\
9900 \pm 200 \\
9650 \\
9230 \pm 70 \\
5200\end{array}$ & $\begin{array}{l}30 \mathrm{v} . \\
11 \mathrm{c} . \\
9 \mathrm{c} . \\
30 \mathrm{v} . \\
10 \mathrm{c} . \\
4 \mathrm{vc} . \\
2 \mathrm{cv} .\end{array}$ \\
\hline CHIII & $\begin{array}{l}\text { E3 } \\
\text { E2 } \\
\text { E1 } \\
\text { D } \\
\text { C }\end{array}$ & 3400 & $\begin{array}{c}\text { Precerámico Temprano } \\
\text { Precerámico Tardío } \\
\text { Formativo T. } \\
\text { Tardío } \\
\text { Tardío }\end{array}$ & $\begin{array}{l}10200 \pm 420 \\
9340 \pm 120 \\
8420 \pm 530 \\
3400 \\
1560\end{array}$ & $\begin{array}{l}2 \mathrm{v} . \\
1 \mathrm{c} . \\
1 \mathrm{c} . \\
4 \mathrm{c} . \\
14 \mathrm{c} . \\
4 \mathrm{v} . \\
19 \mathrm{c} . \\
10 \mathrm{v} . \\
1 \mathrm{c} . \\
3 \mathrm{t} . \\
4 \mathrm{c} .\end{array}$ \\
\hline & B & & Post. & & \\
\hline L.H. & B1 & & Temprano & 9000 & $7 \mathrm{v}$. \\
\hline ICC5 & $\begin{array}{l}10-20 \\
20-10\end{array}$ & & $\begin{array}{l}\text { Tardío } \\
\text { Tardío }\end{array}$ & & $\begin{array}{l}2 \mathrm{v} . \\
1 \mathrm{v} .\end{array}$ \\
\hline ICC7 & & & P. Tardío & 4080 & $\begin{array}{l}1 \mathrm{v} . \\
3 \mathrm{c} .\end{array}$ \\
\hline ICA 1 & $\begin{array}{l}1 \mathrm{~b} \\
1 \mathrm{a}\end{array}$ & & $\begin{array}{l}\text { Tardío } \\
\text { Tardío }\end{array}$ & & $\begin{array}{l}2 \mathrm{v} . \\
4 \mathrm{v} .\end{array}$ \\
\hline QS3 & $\begin{array}{c}2 \mathrm{~b} 19 \\
2 \mathrm{~b} 18 \\
2 \mathrm{~b} 16 \\
2 \mathrm{~b} 14 \\
2 \mathrm{~b} 13 \\
2 \mathrm{~b} 12 \\
2 \mathrm{~b} 11 \\
2 \mathrm{~b} 10 \\
2 \mathrm{~b} 9 \\
2 \mathrm{~b} 8 \\
2 \mathrm{~b} 7 \\
2 \mathrm{~b} 6 / 7 \\
2 \mathrm{~b} 6 \\
2 \mathrm{~b} 5 \\
2 \mathrm{~b} 4 \\
2 \mathrm{~b} 3 \\
2 \mathrm{~b} 2 \\
2 \mathrm{~b} 1\end{array}$ & 4050 & $\begin{array}{c}\text { Prec. T. } \\
\text { “ } \\
\text { “ } \\
\text { “ } \\
7130 \\
\text { N. Int } \\
\\
\text { N. Sup. } \\
\text { Prec. Tardío } \\
\text { “ }\end{array}$ & $\begin{array}{l}8330 \\
8670 \\
6160\end{array}$ & $\begin{array}{c}1 \mathrm{v} . \\
1 . \\
1 \mathrm{v} . \\
5 \mathrm{v} . \\
8 \mathrm{v} . \\
15 \mathrm{v} . \\
6 \mathrm{v} . \\
8 \mathrm{v} . \\
10 \mathrm{v} . \\
5 \mathrm{v} . \\
3 \mathrm{v} . \\
9 \mathrm{v} . \\
14 \mathrm{v} . \\
12 \mathrm{v} . \\
15 \mathrm{v} . \\
10 \mathrm{v} . \\
9 \mathrm{v} .\end{array}$ \\
\hline
\end{tabular}




\begin{tabular}{|c|c|c|c|c|c|}
\hline Sitio & Capa & Altitud & Ubicación temporal & Fechas & Muestras \\
\hline $\mathrm{MCCH}$ & & & Prec. Tardío & $\begin{array}{l}2750 \text { AP } \\
2460 \text { AP }\end{array}$ & $\begin{array}{l}1 \mathrm{v} . \\
4 \mathrm{c} . \\
4 \mathrm{t.}\end{array}$ \\
\hline $\mathrm{CHCH}$ & & & & $260 \mathrm{AP}$ & $\begin{array}{c}1 \mathrm{o} . \\
11 \mathrm{c} . \\
30 \mathrm{t}\end{array}$ \\
\hline
\end{tabular}

\begin{tabular}{|l|l|l|l|}
\hline ICC4 & Inca Cueva Cueva 4 & Depto. de Humahuaca & Jujuy \\
\hline ICC5 & Inca Cueva Cueva 5 & Depto. de Humahuaca & Jujuy \\
\hline ICC7 & Inca Cueva Cueva 7 & Depto. de Humahuaca & Jujuy \\
\hline ICA1 & Inca Cueva Alero 1 & Depto. de Humahuaca \\
\hline CHCHI & Huachichocana Cueva III & Depto. de Tumbaya & Jujuy \\
\hline CHCV & Huachichocana Cueva V & Depto. de Tumbaya & Jujuy \\
\hline L.H. & León Huasi & Depto. de Tumbaya & Jujuy \\
\hline QS3 & Quebrada Seca 3 & Depto. de Antofagasta de la Sierra & Jatamarca \\
\hline MCCH & Morro del Ciénago Chico & Depto. de Susques & Jujuy \\
\hline CHCH & Chulpa Chayal & Depto. de Susques \\
\hline
\end{tabular}

$\mathrm{V}$ : vellones

C: cordeles

T: tejidos

VC: vellones con cuero

O: ovillos

\section{REFERENCIAS CITADAS}

BREWER, 1992. Zooarchaeology: Method, theory and goals. En Archaeological method and theory, M. Schiffer (Ed.). University of Arizona Press, Tucson y Londres.

BUSTINZA, 1987. VI Convención de Camélidos Americanos. Oruro.

CABRERA, 1957. La vegetación de la Puna argentina. Revista de Investigaciones Agr. 11.

CALLE ESCOBAR, R., 1984. Animal reeding and production of American camelids. Lima.

CASTAÑERA, 1991. La vegetación de la cuenca de Pozuelos. En La reserva de la biosfera Laguna de Pozuelos: Un ecosistema pastoril en los Andes Centrales. PER, Universidad Nacional de Jujuy, San Salvador de Jujuy.

DARWIN, C., 1859. On the origin of species by means of natural selection or the preservation of favored races in the struggle for life. Murray, Londres.

DUNNEL, R., 1980. Evolutionary theory and archaeology. En Advances in archaeology method and theory, M. Schiffer (Ed.). Academic Press.

ELKIN, D. y otros, 1992. Avances en el estudio arqueológico de los camélidos del Noroeste Argentino. Actas de la VII Convención Internacional de Especialistas en Camélidos Americanos. San Salvador de Jujuy.
GARCIA FERNANDEZ y R. TECCHI, 1991. Economía y medio ambiente: Análisis de los factores que impiden la expansión de la cría de llamas en la Provincia de Jujuy. En La reserva de la biosfera Laguna de Pozuelos: Un ecosistema pastoril en los Andes Centrales. PER, Universidad Nacional de Jujuy, San Salvador de Jujuy.

MAQUERA LLANO, 1991. Caracterización y persistencia fenotípica en llamas kara y lanudas del Centro Experimental La Raya, Puno. UNA, La Molina.

O'BRIEN, M. y HOLLAND, 1990. Variation, selection and archaeological record. En Archaeological method and theory, M. Schiffer (Ed.). University of Arizona Press, Tucson.

PERKINS, 1973. The beginning of animal domestication in the Near East. American Journal of Archaeology.

REIGADAS, M. C., 1991Ms. Análisis de fibras y cueros de camélidos para la determinación de la domesticación y pastoreo en la Puna Argentina. Informe al CONICET.

1992. La punta del ovillo: Determinación de domesticación y pastoreo a partir del análisis microscópico de fibras y folículos pilosos de camélidos. Arqueología 2.

1993. El beneficio de la lana. Variabilidad tecnológica en manufacturas textiles actuales y arqueológicas en Susques (Provincia de Jujuy). Volumen 25 Aniversario Instituto E. Casanova. 
- 1994Ms. Las fibras como indicador temprano de control cultural de camélidos en el NOA.

- 1995. Criterios metodológicos para la detección del proceso de domesticación en el NOA a partir del análisis de fibras arqueológicas. Cuadernos Revista de la Facultad de Humanidades y Ciencias Sociales.

RUTHSANTZ y C. MOVIA, , 1975. Relevamiento de las estepas andinas del noroeste de la Provincia de Jujuy.

SIMMONS, I., 1982. Biogeografía natural y cultural. Omega, Barcelona.
SOLBRIG, O., 1991. From genes to ecosystems. A research agenda for biodiversity. IUBS, Cambridge.

VIDAL, 1967. La crianza de llamas y algunas características de sus fibras. La Molina.

YACOBACCIO, H., 1991. Información actual, analogía e interpretación del registro arqueológico. X Congreso Nacional de Arqueología Argentina vol. 1. 